\title{
Interview with Agostino Di Scipio
}

\author{
Hans Roels \\ Orpheus Research Centre in Music
}

Agostino Di Scipio (b. Italy, 1962) is a composer, sound artist, music theorist, and scholar. Live computer music, solo or in combination with acoustic instruments, forms a large part of his artistic oeuvre. He has also developed sound installations and large-scale music theatre works. In the last ten years, the interaction between sound, performance space, technology, and performer has become central to his work. The live electronics react to the acoustic characteristics of the hall or to unexpected sounds and, in their turn, change the sound in that hall. This feedback loop between human, technology, and environment is an essential part of what he calls the ecosystemic approach. Di Scipio has written articles on music technology, composition, and social issues in music for journals such as Journal of New Music Research, Computer Music Journal, Contemporary Music Review, Leonardo, Perspectives of New Music, Organised Sound, and Positionen.

Di Scipio visited the Orpheus Institute in February 2012. He gave a lecture-performance during which he performed parts of his solo live-electronics composition Feedback Study and a new work for flute and electronics.

HANS ROELS: Last night your new composition for flute and electronics, 2 pezzi di ascolto e sorveglianza (Two pieces of listening and surveillance), was performed at the Orpheus Institute. It was a try-out session during your lecture-performance. It seems that the creative process of this work took a lot of time. Can you tell us something about this phase?

AGOSTINO DI SCIPIO: Usually I don't start working on a piece with a very clear idea of what I'm going to achieve. However, in this case I had at least the idea to explore a space that is smaller, more individual, and more characteristic than the usual concert hall. I imagined the flute to be a small corridor or a tunnel surrounded by the space of the outer room. Technically I viewed it as a "waveguide." This image of a space within a space, or a niche within a larger environment, had already been an inspiration for me in other recent pieces, for example in installations like Stanze private (2007) and Condotte pubbliche (2011). For this new work, I wanted to do something with a flute. A friend gave me one of his flutes, actually the one that I was using yesterday. For three years I have lived with it and learned how to play it a little bit. I can even play a normal C 
scale! (laughs). But more importantly, I have learned things about the instrument that I previously didn't know. I didn't become a flautist myself, but I did engage with the flute. Next, I started experimenting with microphone placement, inside and outside the instrument. I did this step by step. Already in the beginning I thought it would be good to explore to what extent the hands and fingers could control unwanted sounds, the tiny residual noises due to the key mechanics and to the contact of the hands holding the instrument. When you handle the instrument there is always some noise. Of course, that also depends on the quality of the flute and mine wasn't a very good one. Anyway, I put these and other observations together but I didn't know precisely what I was going to do. I did know, though, that I wanted to use these findings and observations. For this composition, it was a question of finding the proper sequence of performance actions. I spent a lot of time trying out different actions and writing an action score. This was all happening within the context of electro-acoustic amplification and computer processing. As I have said, I was living with the instrument, in fact not only with the flute but with the whole electro-acoustic set-up. Every now and then I went back to this set-up and refined it, and sometimes tried the performance set-up in informal presentations, such as last night. So the piece is worked out through a series of avant-premiere performances ... Until recently, I did not have a deadline to finish this work but now I have one: in September 2012 the work should be more or less finished. I'll hand it over to a real flautist for the official premiere. ${ }^{1}$

ROELS: Is this way of working exceptional for you?

DI SCIPIO: Well, with this flute piece I have spent more time working with the instrument and the set-up than I usually do. Generally I try to design the interactions among the system components, including the instrument and the performer, and that always requires time, of course. Concerning instruments, I try to find someone who has the instrument and can lend it to me, or I buy one. For example, in the next few months I'll be working on a bowing piece, so I purchased a violin, and now I am experimenting with it in a context that is roughly similar to the technical performance set-up. I can then start designing and refining the performance ecosystem, meaning the web of interactions among the system components, including the surrounding space.

ROELS: Room-dependent signal processing often recurs in your work (Di Scipio 2002). Does this imply that you almost necessarily need to experiment?

DI SCIPIO: It does. Now I know, based on experience, that if I stick to a certain kind of relationship, I can expect a certain range of system behaviours although I can't exactly predict what kind of system behaviour will take place and how the performance will evolve. My predictions may be right in some aspects, and totally wrong in others. When you move from the studio to a particular performance space, too many factors change and playing safe becomes impossible.

1 The performance took place at the Fondazione Scelsi, Rome, on 20 September 2012. Manuel Zurria was the flautist. CD, track 4, offers a performance of di Scipio's 2 pezzi di ascolto e sorveglianza. 
ROELS: Your music often involves a certain amount of risk for the performer and for the listener because the performance environment plays an important role and pushes the performance in unexpected directions.

DI SCIPIO: Everybody is at risk in my music (laughs). I call it the fragility of my compositions. As a listener you can experience this fragility, you can hear it. In the case of a strong resonator like a flute, we know in advance that there will be some sound to process. But room-dependent pieces are more subtle and risky, because you never know how the acoustics will be at the moment of the actual performance due to the audience and other circumstances. I take risks and I try to share them with listeners. I try to turn [these risks] into a tangible element in the piece. When unexpected things happen, the system is expected to manifest itself to be really performative, in the normal use of this word-it should work well, stay safe, and keep on going, whatever happens in its surroundings. Before you start, you do not know if everything will work well. By the time you get some sound, and it evolves in a viable articulation in time, it is performative, it functions. That is a result! The quality of the piece and the quality of performance is another issue. Other criteria arise: How many system states are visited through the performance and how is this mapped onto a variety of timbres and gestures? The more varied the resultant range of gestures and timbres, the better the performance. This is not an aesthetic judgement, this is a systemic judgement.

ROELS: Is there a risk that the system becomes so uncontrollable and repetitive that listeners perceive it as boring?

DI SCIPIO: If failure happens, it must be experienced as such. As a composer you are in a position to share the experience of failing. So if you are able to design the sonic process in a way that a failure is communicated and is shared with the audience, then that is a success, it's a good thing to happen. You are not depicting or representing failure, you are witnessing it, experiencing it. Not being able to do anything is a quite interesting experience to have. Also for the listener: you feel that something slips out of your hands. That's the first part of the answer. The second is that I usually provide rules and suggestions in the score to govern the drift, or unwanted repetitive behaviours. The performerwhether on an electronic or acoustic instrument-faces an emergency situation and can take security measures, actions to cope with these situations. In these compositions there is a kind of dramaturgy that is not written or represented, but that is produced and experienced during the performance.

ROELS: In my own experimentation outside the concert hall, these failures do happen, and I guess they are a part of the creative process.

DI SCIPIO: Of course. I know in advance which compositions are more or less risky or fragile. Background Noise Study (2005) is very risky, for example (Di Scipio 2011). Yesterday, as I was rehearsing at the Orpheus Institute, I realised the lecture space wasn't responsive enough. The variety of ambient noise was low, so I preferred not to take the risk of performing it. More generally, there is an inverse relationship between the amount of risk and of preparation time. The more time you have for practising, the less risky the performance becomes. The more time you stay there and live in the environment where you are per- 
forming, the better. You get a feeling of the local acoustics and develop a sensibility for possible performances. This is a problem because you need to ask for longer rehearsal time, which may not always be available.

ROELS: What role does musicianship play in those of your performances that rely heavily on computer processing and other technologies?

DI SCIPIO: I assume that a large part of what we usually mean by "musicianship," especially as experienced by instrumentalists, is about being able to achieve and experience a good balance of means (instrumental action, performance techniques) and ends (expressiveness, quality of sound, capability to interact with others, a sensibility for short-time causal relationships, and so on). I view this as a particular contribution of musicians to society: they balance means and ends and don't let the means command or dictate the ends. Also related to this is the special sensibility of musicians to the surrounding space: instrumental performance is always adapted to the room where it takes place. This is again of the highest relevance in a world where our daily experience is more and more detached from the experience of real spaces and that is ideologically driven by a simplistic notion of technology. I think of my work as focusing strictly on these few grains of musical culture that we are losing because of cultural situations and industrial popular culture (Anderson 2005; Di Scipio 1998).

ROELS: Did you have unexpected reactions from the audience in situations where you felt that they were expecting the normal relationship between means and ends?

DI SCIPIO: I have had some odd reactions. For example, some people question why the audible result should be understood in terms of the emergent properties of the system. Other people don't want to know about the technicalities of the exchange with the environment, they just want to enjoy the result. But if there is any contribution of an artwork to society, it has to do with trying to share. A listener expecting certain results simply doesn't listen to my music, which is about interactions, connections, relationships, shared responsibilities. I can't say how it happened to be so, but my works often question the listener, they ask questions of the listener. Take my installations as an example. ${ }^{2}$ If a visitor-listener talks too loud, the installation remains silent. The idea is that if you came to listen to the work, you should try to be silent and listen to it. There is an ambivalent relationship: the presence of the listener affects the sound that he or she is listening to, the work enables the visitor to reflect on him- or herself as being audibly present in a non-neutral way, and it makes the visitor listen to him- or herself. This is engaging for some people and annoying or too demanding for others. But I don't mind too much about the latter. Actually, when people tell me they are annoyed with this behaviour, I consider this a confirmation that my installation is working! Not because I want to annoy them, but because I want them to feel who they are. My work questions their role as a listener.

2 Untitled 2005, (DAAD Galerie, Berlin, 17 June-3 July 2005); Condotte Pubbliche (Public Conduits)—Ecosystemic Sound Construction (GMM Galerie, Berlin, 19 March-21 May 2011). 
ROELS: As a composer and sound artist you haven't only worked in the concert hall. You have created several theatre compositions and have produced audio installations in musea and other spaces outside the concert hall. Do these spaces give you extra opportunities to experiment?

DISCIPIO: Absolutely, yes. The installations are a special way to focus on certain experiences that remain implicit in concert pieces. They allow me to focus on the physical presence of visitors in the space. There is no necessary sense of dramaturgy, at least not in a short span of time. If you leave the formal concert setting, you can focus on other levels of sonic communication: sound can be a medium for sharing aspects of human experience that are neglected in the concert hall. For example, in installations I am quite free to show the technicalities as they are, and not hide anything in the technical set-up: not because I want to exhibit the technical gear as such, but because I want to stress how sound comes into existence, how it is part of material processes and is shared. The technical element can be overt and clear, so visitors can start thinking about the connections and interactions that produce these sounds. In a concert setting, you cannot highlight this aspect. Theatre is another direction to move in for me, although at this moment I have only composed two or three theatre works. But even a piece like Background Noise Study, in the Vocal Tract, has a kind of theatrical element to it. A performer has a miniature microphone in his/her mouth and uses the mouth as a resonator. I realise that some performance practices that are necessary to produce sound lend themselves quite well to theatrical designs; I am working on a couple of ideas in this direction, but it takes time, especially when non-musicians are involved. On the one hand, the communication with them is problematic, but on the other, the collaboration can be really positive and far-reaching, because they are more free from specific professional expectations and even more available in terms of listening discipline. It's basically the same problem as with non-conventional, non-formal venues and situations, such as courts and open spaces. These require more experimentation. By the way, one objection that was raised against my works is that they don't work in open spaces, because reflected sound is essential in my music. But I can succeed in using spaces, I know how to move my ecosystemic concept to the open air: it just needs more complicated practical arrangements.

ROELS: I can imagine that in an open air situation you have the most openminded audience. The expectation of a certain kind of music is almost absent.

DI SCIPIO: Yes, normally the questions are not on an aesthetic or language level. The crucial element for both expert and non-expert listeners is the awareness they have about what the sound is bringing to them. They can be very active listeners and very engaged, very committed to music, but they may not be able to listen to what sound is bringing to them from the source or the environment. They only enjoy it aesthetically. That's the main problem. Enjoying only aesthetically means that you lend yourself quite well to the industry and industrially produced music. I don't argue for or against this music, but as a composer the problem is that they don't listen to the sound, they only listen to the musical language. 
ROELS: Is this also a motivation to develop your own tools and algorithms? I guess that an important part of your time while composing music and experimenting with the set-up is spent on designing software tools?

DI SCIPIO: Yes, as far as possible I try to be the author and designer of the composition tools and the performance set-up. For me it is very important to be responsible for what I present in public. It is a kind of testimony and political statement to be responsible for your actions. It also means to be competent in technical areas and to be aware of the musical meaning of a composition and its performance. Such an approach acts as a mirror for the audience and that is why it questions the listener.

ROELS: How important is your independence? You have your own personal studio but you have also been a guest at several art and research institutions.

DI SCIPIO: In part, this independence happened probably because of my bad character, but after a while it became a prerequisite to do things that are impossible within larger institutes because they have very different expectations. Research funding is flowing in this or that direction and you have to keep up with it. It is the basic dialectic of the researcher and the artist within the academy. Using very simple technical configurations is also very important to me. I prefer to design and work on the interrelationships between simple processes, between tools that are adaptable and not too specific. I try to avoid creating works that need a specialised, powerful piece of gear or a computationally expensive device. I don't raise money to buy hi-tech tools, or to rent special studios and rooms. I try to do my best with the little that I can personally afford. Some people have visited me in my studio and been surprised to see how basic my studio configuration is. They probably expected many powerful computers, many screens, and many speakers. Flexibility in the studio is far more important for me, the possibility to pack and unpack, to try a set-up, and then move to a different one with a certain ease. The overall configurations are capable of being rewired and can be tested and dismantled quite easily, although not necessarily quickly.

ROELS: Leaving empty spaces in your studio or workshop gives you the opportunity to change plans and experiments while you are composing. You can try something new if you suddenly want to.

DI SCIPIO: Flexibility in the technical configurations in the studio has to do with the creative process, that is true. Setting up things and materials in an empty space allows you to focus on the system relationships you are designing, to make them work on their own, leaving aside what is unnecessary. You can draw a profile or a spatial horizon within which the work performs the way it does. By the way, the latter point brings us to a related issue. Installations have a temporal horizon, a duration within which the listener pays attention to the installation, for example five or ten minutes or maybe even twenty depending on who is listening. But there is also a spatial horizon, which is how far you can go from the installation and still witness what it is doing. This spatial horizon is a very important element of musical form. We think of form only in terms of dramaturgy and time but it also relates to space. Form exists within a certain sphere and within a certain horizon. There is an ecological approach to psy- 
choacoustics that is very valuable in my opinion. It concerns the perception of space, movement in space, presence, bodily presence, and proximity (Neuhoff 2004; Rocchesso and Fontana 2003).

\section{References}

Anderson, Christine, and Agostino Di Scipio. 2005. "Dynamic Networks of Sonic Interactions: An Interview with Agostino Di Scipio." Computer Music Journal 29 (3): 11-28.

Di Scipio, Agostino. 1998. "Questions Concerning Music Technology." Angelaki: Journal of the Theoretical Humanities 3 (2): $31-40$.

- 2003. "'Sound Is the Interface':

From Interactive to Ecosystemic Signal

Processing." Organised Sound 8 (3): 269-77.

- — . 2011. "Listening to Yourself through the Otherself: On Background Noise Study and Other Works." Organised Sound 16 (2): 97-108.

Neuhoff, John G., ed. 2004. Ecological Psychoacoustics. Amsterdam: Elsevier Academic Press.

Rocchesso, Davide, and Federico Fontana, eds. 2003. The Sounding Object. Florence: Mondo estremo. 
